\title{
Comparativo entre a distribuição dos tempos na produção com índices de produtividade para o serviço de montagem de fôrma de madeira
}

\section{Comparing between the distribution of time in production with productivity indices for the} formwork assembly

\author{
B. A. Souza ; D. de G. Santos \\ IPrograma de pós-graduação em Engenharia Civil, Universidade Federal de Sergipe, 49100-000, São Cristovão - \\ Sergipe, Brasil
}

*bralmeidae@ymail.com

(Recebido em 10 de junho de 2015; aceito em 04 de novembro de 2015)

\begin{abstract}
A mão de obra é um dos principais recursos para qualquer setor da indústria, não sendo diferente para a construção civil. Devido a sua importância, deve-se buscar que a mão de obra seja cada vez mais eficiente melhorando o seu índice de produtividade. Diante deste cenário o objetivo desta pesquisa é o comparativo entre os índices de produtividade para execução de fôrmas de madeira para estruturas de concreto, encontrados em tabelas e ou sistemas de preços, com o índice utilizado no orçamento da obra, utilizando ainda a técnica de amostragem do trabalho. Na metodologia, o primeiro passo foi conhecer o próprio índice de produtividade, aferindo-o em campo. É importante também que não só o calcule como também conheça os fatores que influenciam em sua melhora ou piora. Esta análise pode ser feita através de pesquisas de amostragem do trabalho. Como resultado, levantados os índices foi realizada uma comparação entre a equipe presente na obra, com a equipe teórica calcula através desses índices. Ao final foi feita uma pesquisa de amostragem do trabalho na busca de obter informações que mostrassem possíveis pontos de melhora nos processos para melhorar o índice de produtividade dessa mão de obra.

Palavras-chave: produtividade, amostragem do trabalho
\end{abstract}

The workforce is a key resource for any industry sector, no different for the construction industry. Due to its importance, must be sought that the workforce is becoming more efficient by improving their productivity index. In this scenario the objective of this research is the comparison of the productivity indices for wooden formwork of execution for concrete structures found in tables and or pricing systems, with the index used in the budget of the work, still using the sampling technique from work. In the methodology, the first step was to identify the actual productivity index, assessing it in the field. It is also important that not only calculate but also know the factors that influence its improvement or worsening. This analysis can be done by sampling research work. As a result, raised the rates was made a comparison between this team's work, with the theoretical team calculates through these indices. At the end of a working sample survey was conducted in search of information that showed possible points of improvement in processes to improve productivity rate this manpower.

Keywords: productivity, sampling work

\section{INTRODUÇÃO}

O setor da construção civil representa grande participação no Produto Interno Bruto (PIB) nacional [1]. Para gerar tal riqueza, são necessários vários recursos com destaque para as matérias primas e a mão de obra. Em comparação com a indústria seriada (tradicional), percebese que a quantidade de mão de obra necessária é mais elevada, mostrando o quão importante é estudar e investir na produtividade desse setor.

A importância do estudo do índice de produtividade se dá na construção do histórico da empresa, onde serão estabelecidos parâmetros. Esses poderão ser comparados com os de outras empresas do setor, a fim de estimar a capacidade competitiva. Para Souza (2006) [1], o estudo da produtividade formará também uma base de dados, onde esses poderão ser utilizados para uma futura tomada de decisão da empresa, além de auxiliar no planejamento de obras futuras e facilitar no estabelecimento de metas. 
Tradicionalmente, estuda-se o índice de produtividade e os fatores que interferem na produtividade para promover melhorias na produção. Porém, verifica-se que existem muitos fatores que interferem neste resultado e que ocorrem na forma de perdas de tempo das atividades de produção.

A mão de obra é algo dimensionável a partir de índices. Essa não pode ser dimensionada arbitrariamente, a cargo da própria equipe ou de seu supervisor. Essa iniciativa contribui para evitar perdas referentes à mão de obra [3].

A mão de obra é um dos principais custos inerentes à construção de um empreendimento. Este precisa ser bem administrado, pois influenciará diretamente nos lucros de uma empresa. Pode-se aumentar a produtividade dessa mão de obra utilizando algumas técnicas, mas também é importante que se diminuam as perdas inerentes a ela.

Para Santos (1995) [4], aumentar a produtividade é mais do que introduzir equipamentos, é preciso criar um sistema capaz de interpretar a complexa teia de relacionamento entre a técnica e o fator humano, de modo a aperfeiçoar todo o processo, não apenas uma parte dele.

A implementação de qualquer sistema requer treinamento das pessoas envolvidas para conhecer o sistema e utilizá-lo de modo mais eficiente. Porém, algumas empresas do setor construção civil não dão tanta importância para o treinamento de sua mão de obra, o que gera uma baixa qualidade na execução do serviço e uma alta rotatividade, resultando também em diminuição da produtividade. Além disso, a perda elevada passa a ser vista como normal [5].

Koskela (1992) [6] iniciou o estudo de perdas, de uma forma mais ampla, na construção civil, ao apresentar uma nova filosofia de produção (Construção Enxuta). A partir deste estudo, várias pesquisas foram desenvolvidas por Koskela e por diversos pesquisadores, para divulgar e implementar na prática a Construção Enxuta. O enfoque desse estudo não é só as atividades de conversão mas também as atividades de fluxo. Essas são atividades pertencentes ao ciclo de produção e que não agregam valor ao produto final [6].

Koskela (1992) destaca que se deve procurar otimizar as atividades que agregam valor e reduzir ao máximo as atividades que não agregam. As atividades que não agregam valor não podem ser completamente eliminadas, visto que algumas dessas atividades como o planejamento, contabilidade e a prevenção de acidentes são necessárias e agregam valor ao cliente interno.

Observa-se um aumento da produtividade da mão de obra da Construção Civil quando são aplicados os 11 princípios para redução de perdas propostos por Koskela (1992). Isto acontece porque a aplicação dos princípios ocorre tanto nas atividades que agregam valor (atividades produtivas) como nas que não agregam valor (atividades improdutivas e auxiliares) e isto influencia o rendimento do trabalho. Há um consenso entre os estudiosos de que o objetivo principal da construção enxuta é criar valor para os clientes, melhorar as operações gradativamente e com foco em reduzir perdas [7].

Ao estudar as atividades de conversão e de fluxo, observa-se que as primeiras são formadas por atividades de processamento, que comumente agregam valor ao produto e são conhecidas do gerenciamento tradicional. Para essas atividades o principal índice de desempenho é a produtividade. Por sua vez, as atividades de fluxo não agregam valor ao produto [8].

Koskela (2000) [9] preocupou-se com os dados de entrada para a realização de determinado processo, sendo estes denominados de sete fluxos, que devem se unir para o cumprimento de uma tarefa, em termos de suas atividades componentes. Os sete fluxos são: projetos (informações), materiais/componentes, mão de obra, equipamentos, espaço, tarefas pré-requisito e condições externas.

Shingo (1996) [10] classifica as perdas na produção em sete tipos: superprodução, espera, transporte, processamento, movimento, produção de produtos defeituosos e estoques. Um oitavo tipo de é denominado making-do [11], que ocorre quando um operário começa um processo sem que todos os pré-requisitos estejam concluídos ou disponíveis. Esse início no tempo errado pode gerar um aumento no tempo do processamento e um aumento da variabilidade do produto [11].

É sabido ainda que a continuidade do trabalho relaciona-se diretamente com o acréscimo de tempo. Neste sentido, para investigar a perda de tempo, Kalsaas (2010) [12] abordou dentre outros aspectos a produtividade, dividindo o tempo dedicado ao trabalho em três grupos: o trabalho que adiciona valor, a preparação para o trabalho e a perda em si. Como resultado, 
foram observadas interrupções/fragmentações desnecessárias do trabalho, devido à falta de materiais e equipamentos, e falhas na execução, que resultam em retrabalho, e, por sua vez, no making-do [11].

Segundo Kalsaas (2010) [12], transporte, espera e movimentação desnecessária contém aspectos óbvios e podem ser medidos em termos de tempo. Apesar de óbvias, assim como a superprodução, estas ações resultam em perdas e acontecem comumente na construção civil. A despeito das diferenças de país, região e empresa, conforme citado pelo autor, verifica-se que as dificuldades e os exemplos de uso do making-do são comuns a qualquer realidade da construção civil, como também da indústria tradicional [13].

Vieira (2006) [14] afirma que o desempenho operacional da construção civil no Brasil não acompanhou a evolução que foi sentida nos demais setores da indústria, sendo comum a perda e a improvisação dentro do canteiro de obras. Costa e Santos (2011) [15] ainda destacam que a mão de obra que tem acompanhado o setor é pouco qualificada, o que dificulta o desenvolvimento do setor.

Assim, outra forma de contribuir com melhorias relacionadas à tarefa realizada pelos operários, que refletem na produtividade da equipe, é o estudo da distribuição das tarefas no tempo, ou seja, durante a jornada de trabalho. Isto está diretamente relacionado com o dimensionamento da equipe e como a mesma utiliza o tempo para realizar as tarefas programadas. Este estudo é denominado de amostragem do trabalho. Nesta técnica, as atividades são distribuídas ao longo do tempo em atividades produtivas, auxiliares e improdutivas. As primeiras são as que agregam valor ao produto, os dois outros tipos não agregam valor ao produto, conforme mencionado anteriormente.

Nascimento (2013) [16] destaca que essa quantificação dos tempos é importante para o entendimento dos fatores que influenciam na produção. Uma vez conhecido os principais motivos que geram improdutividade das equipes, devem ser tomadas atitudes gerenciais para que seja possível mitigar tais perdas, desde que as soluções sejam economicamente viáveis e não comprometam outras atividades do processo.

Para Carvalho et al. (2004) [17], uma tendência que vem ocorrendo em diversos países do mundo é como medir e aumentar a produtividade da mão de obra, sendo esta uma preocupação atual. Para isso, Freitas et al. (1994) apud Carvalho et al. (2004) [17] afirmam que "somente através da observação do trabalho e do levantamento de dados é que podem ser propostas modificações no processo produtivo e explicadas estas modificações aos operários".

O método mais utilizado é o método de amostragem do trabalho [4]. O método consiste na observação e no registro do que o operário está executando, no exato momento em que é observado, em planilhas pré-elaboradas, contendo todas as atividades constantes do ciclo da execução do serviço [5]. Para as observações, adotam-se intervalos regulares de tempo entre elas (tempo de ciclo), disciplinando o trabalho do observador.

Para este método é necessário calcular um número mínimo de observações para cada nível de confiabilidade desejado. De acordo com Carvalho et al. (2004) [17], é possível calcular o número de observações a partir da equação (1) oriunda de métodos estatísticos.

$$
N=4 \cdot \frac{p \cdot(1-p)}{(S \cdot p)^{2}}
$$

Onde:

$\mathrm{N}$ é o número de observações a serem realizadas;

$\mathrm{S}$ é o erro relativo (\%);

p é o percentual de ocorrência do evento, em decimal.

O nível de confiabilidade geralmente adotado é de $95 \%$. Sendo assim, o erro relativo é de $5 \%$ [17]. A variável "p" representa o percentual de ocorrência do evento. Esse é um valor obtido apenas ao término da pesquisa. Na construção civil, geralmente os tempos produtivos são de $33 \%$ e os outros $67 \%$ estão divididos entre auxiliares e improdutivos [4]. 
A técnica de amostragem do trabalho é uma ferramenta importante para que as empresas possam fazer o estudo dos tempos gastos em cada um dos três tipos de atividades.

As atividades produtivas são as atividades que são indispensáveis, que de fato agregam valor ao produto. Existem ainda as atividades complementares, ou auxiliares, que não agregam valor, mas que são necessárias para a execução do serviço. As atividades improdutivas existem e devem ser eliminadas o máximo possível no processo produtivo, sem comprometer outras atividades.

O objetivo da pesquisa foi comparar os índices de produtividade para as fôrmas encontrados em tabelas e ou sistemas de preços com o índice utilizado no orçamento da obra, utilizando ainda a técnica de amostragem do trabalho.

\section{MATERIAL E MÉTODOS}

O canteiro de obras pesquisado possui duas equipes de carpintaria, uma delas é composta por mão de obra própria e a outra por mão de obra terceirizada. Porém, optou-se por pesquisar o trabalho desenvolvido pela equipe própria, para contribuir com o banco de dados de índice de produtividade da empresa.

A equipe de carpintaria possui um conjunto completo de fôrma. Esse conjunto contempla as fôrmas dos pilares, fundo e laterais de vigas, assoalho e escoramento. Além do conjunto completo, a equipe utilizou como instrumento de trabalho um conjunto de fundo de vigas e escoras extra. Esta foi uma estratégia tomada pela empresa para tornar mais rápida a execução da montagem da fôrma. A fôrma foi confeccionada na própria obra através dos projetos estruturais.

O transporte dos materiais da carpintaria era feito pelos próprios operários manualmente, ou seja, não foi utilizado nenhum tipo de equipamento mecânico.

Em termos das ferramentas de pesquisa aplicadas, para proceder ao acompanhamento dos serviços em termos dos tempos de produção, foi adotada a técnica de amostragem do trabalho, para que fosse feito o registro em planilha pré-elaborada da atividade executada pelo operário no momento da observação, e foi calculado o índice de produtividade.

Foi realizada uma pesquisa através da internet, a fim de obter os índices de produtividade presentes em tabelas e ou sistemas de composição de preços, como Orçamento de Obras Sergipe (ORSE) e Sistema Nacional de Preços e Índices (SINAPI).

Foi adotado como sendo o índice de produtividade, da obra em estudo, o índice que estava contido no orçamento da mesma. Este índice foi obtido juntamente ao setor de orçamento da empresa.

Procedeu-se ainda a uma comparação entre dados teóricos e de campo quanto ao índice de produtividade e o dimensionamento da equipe. É sabido que o índice de produtividade (Ip) é calculado pela equação (2):

$$
I p=\frac{H \cdot h}{Q}
$$

Onde:

H é a quantidade de operários na equipe;

$\mathrm{h}$ é a quantidade de tempo para realizar a tarefa, em horas;

$\mathrm{Q}$ é a quantidade de trabalho a ser realizado $\left(\mathrm{m}^{2}, \mathrm{~m}^{3}, \mathrm{~kg}\right.$ etc. $)$.

No caso do dimensionamento da equipe, utiliza-se a equação (2), inserindo o valor do índice de produtividade, para buscar a variável $\mathrm{H}$.

É necessário calcular um número mínimo de observações (N) para cada nível de confiabilidade desejado, conforme apresentado [17].

Quanto à amostragem do trabalho, esta técnica consiste na observação e no registro do que o operário está executando, no exato momento em que é observado, em planilhas pré-elaboradas, contendo todas as atividades constantes do ciclo da execução do serviço [5]. Para as 
observações, adotam-se intervalos regulares de tempo entre elas (tempo de ciclo), disciplinando o trabalho do observador. Adotou-se ainda um erro de 10,00\% e foram necessárias 2437 observações. Esta quantidade de observações é válida para medições com um intervalo de tempo de 15 em 15 minutos, durante 5 horas por dia e com uma equipe de 14 operários. Com estes dados, a coleta foi finalizada em 09 dias úteis.

Deste modo, as atividades observadas eram classificadas como produtivas, auxiliares ou improdutivas, resultando ao final do dia no percentual de tempo gasto em cada categoria.

Verifica-se que a classificação das atividades em produtivas, auxiliares e improdutivas é de caráter subjetivo e pessoal para cada pesquisador. Sendo assim, foram elaboradas planilhas por processo construtivo e dentro destes por tipo de mão de obra, se oficial ou ajudante (serventes), considerando o procedimento operacional adotado pela empresa pesquisada. As Figuras 1 e 2 apresentam as atividades desenvolvidas pelos oficiais da carpintaria e as Figuras 3 e 4 as atividades para os ajudantes.

OFICIAIS CARPINTEIROS
\begin{tabular}{|l|l|l|}
\hline \multicolumn{1}{|c|}{ PRODUTIVAS } & \multicolumn{1}{|c|}{ AUXILIARES } & \multicolumn{1}{c|}{ IMPRODUTIVAS } \\
\hline 101 Colocação do gastalho & 201 Transferência de eixo & 301 Espera por material \\
102 Fixação da grade do fundo & 202 Aprumar grade junto à forma & 302 Espera por conferência do técnico \\
103 Fixação de forma da grade & 203 Marcação da linha de nível & 303 Necessidades fisiológicas \\
104 Fixação de formas laterais & 204 Desforma & 304 Descanso \\
105 Fixação de forma de fechamento & 205 Reforma de forma & 305 Parado devido chuva \\
106 Fixação de grade de fechamento & 206 Nivelamento & 306 Falta \\
107 Colocação de atracadores no pilar & 207 Colocação de garfos & 307 Retrabalho \\
108 Colocação de fundo de vigas & 208 Colocação de escoras & 308 Fora do posto de trabalho \\
109 Colocação e fixação de formas laterais & 209 Receber instruções & 309 Parado sem motivo \\
110 Montagem de assoalho & 210 Confecção de gastalho maluco & 310 Em deslocamento \\
111 Fixação arame painel externo & 211 Fechamento encontro de viga & 311 Transporte de materiais \\
112 Forma rebaixamento de varanda & 212 Amarras nas escoras & \\
\hline & 213 Travamento fundo de viga & \\
\hline
\end{tabular}

Figura 1: Atividades produtivas, auxiliares e improdutivas para os carpinteiros.

Fonte: Elaborado pelo autor.

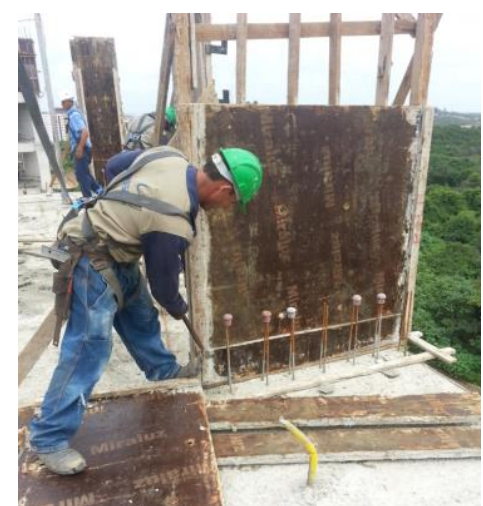

(a)

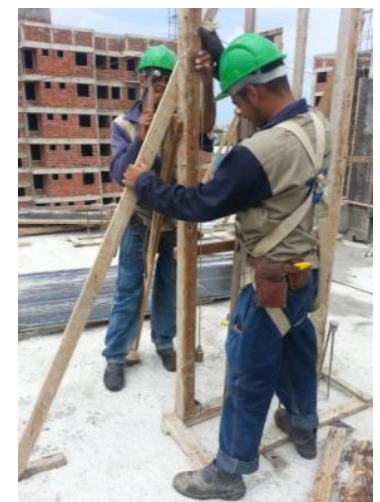

(b)

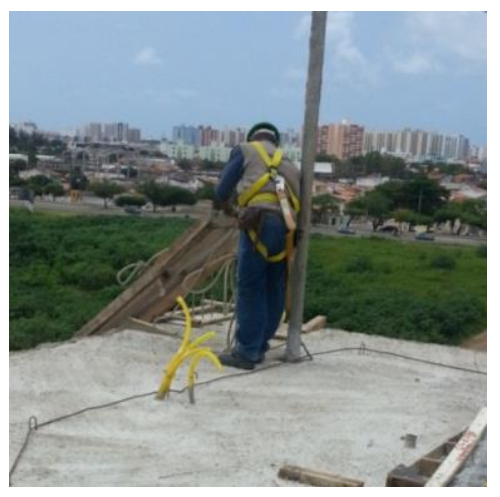

(c)

Figura 2: Exemplo de atividade produtiva (a), auxiliar (b) e improdutiva (c) dos carpinteiros.

Fonte: Elaborado pelo autor. 
SERVENTES

\begin{tabular}{|l|l|l|}
\hline \multicolumn{1}{|c|}{ PRODUTIVAS } & \multicolumn{1}{c|}{ AUXILIARES } & \multicolumn{1}{c|}{ IMPRODUTIVAS } \\
\hline 401 Apicoar concreto base de pilar & 501 Receber instruções & 601 Espera por material \\
402 Passar desmoldante nas formas & 502 Limpeza do posto de trabalho & 602 Necessidades fisiológicas \\
403 Transporte de material/ferramenta & 503 Colocação de grade & 603 Descanso \\
404 Colocação de garfos & & 604 Parado devido chuva \\
405 Colocação de escoras & & 605 Falta \\
406 Limpeza do material & & 606 Retrabalho \\
407 Segurar forma para reposicionamento & & 607 Fora do posto de trabalho \\
408 Preparação de estrutura para desforma & & 608 Parado sem motivo \\
409 Preparação de material & & 609 Parado \\
410 Auxilio gastalho & 610 Em deslocamento \\
\hline
\end{tabular}

Figura 3: Atividades produtivas, auxiliares e improdutivas para os serventes da carpintaria.

Fonte: Elaborado pelo autor.

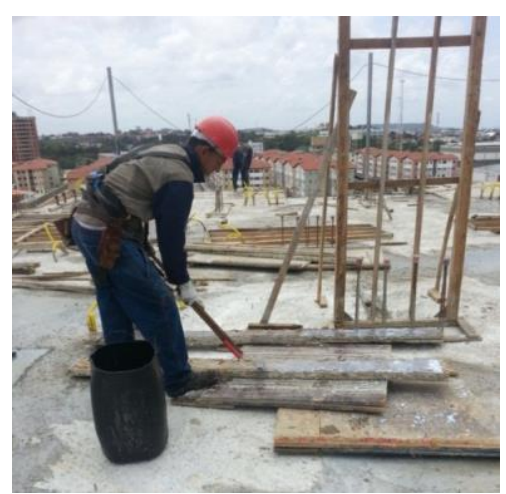

Figura 4: Exemplo de atividade produtiva dos ajudantes.

Fonte: Elaborado pelo autor.

\section{RESULTADOS E DISCUSSÃO}

É sabido que na comparação de índices de produtividade, o indicado seria comparar índices obtidos em circunstâncias semelhantes. Porém, sabe-se que, muitas vezes, os autores não explicitam todas as informações nos levantamentos que realizam. Sendo assim, para esta pesquisa foram pesquisados na literatura índices em situações semelhantes e na ausência destes índices com características próximas. Deste modo, pesquisou-se na literatura, além de trabalhos científicos, dados do ORSE e SINAPI, para o serviço (processo construtivo) em análise. Esse fato mostra a dificuldade das empresas em encontrar composições que retratem a realidade da mesma, o que torna o trabalho de apropriação em campo de índices de produtividade e consumo de material importante para a construção do banco de dados das empresas.

Foi feita então a coleta dos índices, conforme descrito na metodologia, obtendo os seguintes resultados:

\section{-ORSE}

Código: 7557

Descrição: Fôrma plana para lajes, em compensado plastificado de $17 \mathrm{~mm}, 12$ usos, inclusive escoramento.

Índice de produtividade para oficiais: $0,524 \mathrm{Hh} / \mathrm{m}^{2}$.

Índice de produtividade para serventes: $0,131 \mathrm{Hh} / \mathrm{m}^{2}$.

Duração real observada na pesquisa: 7 dias - 63 horas. 
Equipe dimensionada: 11 carpinteiros e 3 serventes.

-SINAPI

Código: 84220

Descrição: Fôrma para estruturas de concreto (pilar, viga e laje) em chapa de madeira compensada plastificada, de $1,10 \times 2,20$, espessura $=12 \mathrm{~mm}, 08$ utilizações (fabricação, montagem e desmontagem - exclusive escoramento).

Índice de produtividade para oficiais: $0,66 \mathrm{Hh} / \mathrm{m}^{2}$.

Índice de produtividade para serventes: $0,16 \mathrm{Hh} / \mathrm{m}^{2}$.

Duração real observada na pesquisa: 7 dias - 63 horas.

Equipe dimensionada: 13 carpinteiros e 3 serventes.

\section{-EMPRESA PESQUISADA}

Descrição: Fôrma plana para lajes, em compensado plastificado, inclusive escoramento. Estavam previstas 12 reutilizações deste material.

Índice de produtividade para oficiais: $0,664 \mathrm{Hh} / \mathrm{m}^{2}$.

Índice de produtividade para serventes: $0,423 \mathrm{Hh} / \mathrm{m}^{2}$.

Duração real observada na pesquisa: 7 dias - 63 horas.

Equipe dimensionada: 13 carpinteiros e 8 servente.

Uma vez conhecida a quantidade do serviço e o tempo total gasto, foi feito o dimensionamento da equipe (Tabela 1) a partir dos índices de produtividade citados, para comparar com a equipe que estava em campo. Pode-se observar que a equipe presente na obra encontra-se bem dimensionada, pois esta apresentava um número menor de oficiais do que o número calculado. Com relação aos profissionais classificados como não oficiais, houve um acréscimo de dois meio oficiais em relação aos índices do ORSE e do SINAPI. Porém, em relação ao índice da própria empresa, apresentou-se em menor quantidade, visto que o índice sugere uma equipe com 8 serventes e a real em obra era de dois meio oficiais e 3 serventes.

Tabela1: Comparativo do dimensionamento da equipe para o serviço de fôrma.

\begin{tabular}{cccc}
\hline & \multicolumn{3}{c}{ Quantidade de operários } \\
\cline { 2 - 4 } Sistemas & Oficiais & Ajudantes & Meio oficiais \\
\hline ORSE & 11 & 3 & - \\
\hline SINAPI & 13 & 3 & - \\
\hline Índice da empresa & 13 & 8 & 2 \\
\hline Equipe real & 10 & 3 &
\end{tabular}

Os índices de produtividade da empresa pesquisada mostraram com valores elevados, comparados aos índices encontrados em fontes como ORSE e SINAPI. A empresa poderia rever esse índice, uma vez que a equipe em campo era menor do que a equipe dimensionada com base nesse índice. Esse índice elevado onera o orçamento da obra de maneira desnecessária, o que pode atrapalhar nas tomadas de decisões, visto que, o custo da obra é um fator importante quanto a sua viabilidade.

Após análise dos dados de amostragem do trabalho e de realizar o acompanhamento dos serviços, foi possível elaborar o quadro apresentado na Tabela 2 e a Figura 5. 
Tabela 2: Resumo das percentagens de tempos produtivos, improdutivos e auxiliares para o serviço de execução de fôrma.

\begin{tabular}{cccc}
\hline & \multicolumn{3}{c}{ Percentual da distribuição dos tempos por classe } \\
\cline { 2 - 4 } & \% produtivos & \% auxiliares & \% improdutivos \\
\hline Oficiais & $33,16 \%$ & $27,97 \%$ & $38,87 \%$ \\
Serventes & $68,85 \%$ & $0,06 \%$ & $31,09 \%$ \\
\hline
\end{tabular}

Fonte: Elaborado pelo autor.

A partir dos resultados diários (Figura 5) pode-se observar que os dois primeiros dias, logo após a concretagem da laje, são dias com alta porcentagem de tempos produtivos. Isso se deve à quantidade de volume de serviço que precisa ser feito. Esses serviços vão desde a colocação do gastalho até a colocação dos fundos e laterais de vigas. No primeiro dia do primeiro ciclo (pavimento 4) as medições foram realizadas à tarde, ou seja, os gastalhos já estavam colocados e grande parte dos carpinteiros estava realizando atividades produtivas. No primeiro dia dos outros dois ciclos (pavimentos 5 e 6), as medições foram feitas pela manhã, diminuindo assim a porcentagem de tempos produtivos, pois apenas dois carpinteiros colocavam os gastalhos enquanto os outros desformavam o pavimento inferior.

No terceiro dia do ciclo, a porcentagem de tempos produtivos diminui e a de tempos improdutivos aumenta. Isso ocorre devido ao fato de todo o transporte de material dos pavimentos inferiores para o atual pavimento ter sido realizado também pelos oficiais. Esses materiais transportados eram as escoras metálicas, as longarinas e as transversinas, que servem de apoio para o assoalho assim como a fôrma do assoalho.

No quarto dia, a porcentagem de tempos produtivos volta a aumentar, pois neste dia ocorreu a montagem de todo o assoalho, terminando com a conclusão do mesmo, ou seja, até que este ficasse pronto para a concretagem dos pilares.

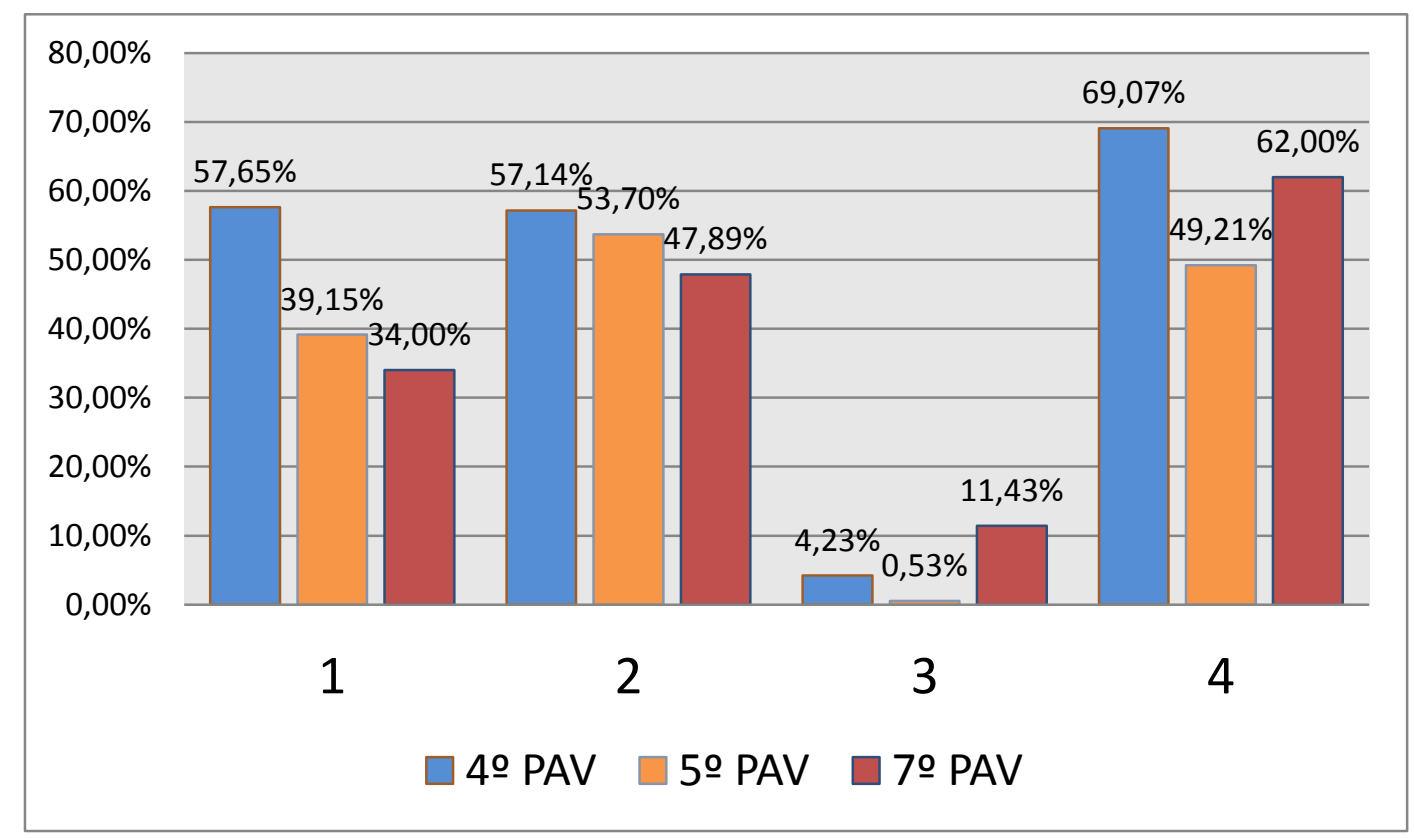

Figura 5: Resumo das percentagens de tempos produtivos (azul),auxiliares (laranja) $e$ improdutivos (vermelho) para o serviço de execução de fôrma para cada dia de trabalho para cada ciclo observado.

Fonte: Elaborado pelo autor. 
A pesquisa de amostragem do trabalho retornou resultados importantes, mostrando que a equipe de montagem de fôrma tem elevados percentuais de tempos improdutivos. Uma redução dos fatores que geram essa improdutividade poderia influenciar numa diminuição ainda maior do índice de produtividade adotado pela da empresa.

\section{CONCLUSÃO}

Observa-se que os motivos dos percentuais de improdutividade das equipes resultam de alterações na maneira de se construir. Quando se minimiza estas situações contribui-se para a diminuição das perdas, o aumento da produtividade das equipes e maior competitividade das empresas construtoras.

Verificou-se ainda que no serviço de fôrma foi possível comparar a distribuição dos tempos com o índice de produtividade real, bem como comparar este índice com os teóricos. Observouse que os dados reais eram mais produtivos do que os disponíveis na literatura ou calculado a partir de dados disponibilizados pela equipe da obra.

Quanto à aplicação da técnica de amostragem do trabalho, foram calculados os percentuais de cada tipo de atividade, resultando em os percentuais para oficiais $(33,16 \% ; 27,97 \%$ e $38,87 \%)$ e ajudantes $(68,85 \% ; 0,06 \%$ e $31,09 \%)$, para as atividades produtivas, auxiliares e improdutivas respectivamente.

Como é possível observar, no serviço de execução de fôrma, a porcentagem de atividades produtivas está próximo do encontrado na literatura e com um aumento no percentual das atividades improdutivas. Esses índices foram consequências de uma elevada observação de atividades que não agregam valor ao carpinteiro (transporte de material) e também devido à baixa produtividade entre os dias da concretagem dos pilares e a concretagem da laje.

\section{REFERÊNCIAS BIBLIOGRÁFICAS}

1. Souza UEL de. Como aumentar a eficiência da mão de obra: manual de gestão da produtividade na construção civil. São Paulo: Editora Pini. 2006.

2. Souza U E L de, Araújo LOC de. Do Construction - Uma abordagem de gestão fundamentada no uso de indicadores. In: Simpósio Brasileiro de Gestão e Economia da Construção, IV, 2005, Porto Alegre. Anais... Porto Alegre. 2005. 9p.

3. Santos A. dos. Método de intervenção em obras de edificações enfocando o sistema de movimentação e armazenamento de materiais: um estudo de caso. 1995, 170p. Dissertação (Mestrado em Engenharia Civil). Curso de Pós-Graduação em Engenharia Civil. Universidade Federal do Rio Grande do Sul. Porto Alegre. 1995.

4. Librelotto LI, Mutti C do N, Oliveira PVH de, Jungles AE. Análise do emprego dos tempos de mão de obra utilizando a técnica de amostragem do trabalho. In: Encontro Nacional de Tecnologia do Ambiente Construído, III, 2000, Salvador. Anais... Salvador. 2000. 8p.

5. Isatto EL, Formoso CT. A nova filosofia de produção e a redução de perdas na construção civil. In: Encontro Nacional de Tecnologia do Ambiente Construído, N, 1998, Florianópolis. Anais... Florianópolis. 1998. 10p.

6. Moura RSLM, Heineck LFM. Linha de Balanço - Síntese dos princípios de produção enxuta aplicados à programação de obras? In: Encontro Nacional de Tecnologia do Ambiente Construído, 2014, Maceió. Anais... Maceio. 2014.

7. Souza B A. Contribuições do estudo dos tempos das atividades nos serviços de estrutura e alvenaria para a redução de perdas na construção. 2013, 78p. Trabalho de Conclusão de Curso. Departamento de Engenharia Civil. Universidade Federal de Sergipe, São Cristóvão. 2013.

8. Koskela L. An exploration towards a production theory and its application to construction. 2000. 298p. Doctor of Philosophy, Helsinki University of Technology, VTT Technical Research Centre of Finland, Espoo. 2000.

9. Shingo S. O sistema Toyota de produção do ponto de vista da engenharia de produção. $2^{a}$ edição. Porto Alegre: Bookman. 1996. 291p.

10. Koskela L. Making-do: the eighth category of waste. In: Conference of the International Group for Lean Construction, 12., 2004, Elsinore, Denmark. Proceedings... Elsinore, 10 p. 2004. Disponível em: http://www.iglc.net. 
11. Kalsaas BT. Work-time waste in construction. In: Conference of the International Group for Lean Construction, 18., 2010, Haifa, Israel. Proceedings... Haifa, Israel, 2010, 11p. Disponível em: http://www.iglc.net.

12. Santos DG, Oliveira FM, Santos Neto AT. Aplicação de atividades facilitadoras no processo construtivo de estruturas: estudo de caso. In: Simpósio Brasileiro de Gestão e Economia da Construção, 7º , Belém, 2011. Anais... Belém, 2011, 13p.

13. Vieira HF. Logística aplicada à construção civil: como melhorar o fluxo de produção nas obras. São Paulo; Editora Pini, 2006, 178p.

14. Costa EBD, Santos ADO. Programas de humanização na construção civil em Maceió. In: Simpósio Brasileiro de Gestão e Economia da Construção, Vii, 2011, Belém. Anais... Belém, 2011.

15. Nascimento $\mathrm{R}$ da $\mathrm{S}$. Fatores que interferem na produtividade dos processos construtivos de contrapiso e revestimento cerâmico e sua relação com as atividades de fluxo do trabalho: Estudo de caso. 2013. Trabalho acadêmico orientado. Departamento de Engenharia Civil. Universidade Federal de Sergipe. São Cristóvão. 2013.

16. Carvalho LO, Heineck LFM, Jungles AE. Uma crítica à utilização da técnica de observações instantâneas para obtenção de tempos produtivos, auxiliares e improdutivos em obra: uma metodologia para obtenção das constantes orçamentárias de consumo dos serviços de construção. In: Encontro Nacional de Tecnologia do Ambiente Construído, X, 2004, São Paulo. Anais... São Paulo, 2004. 